\title{
Linguistic Features for Readability Assessment
}

\author{
Tovly Deutsch Masoud Jasbi Stuart Shieber \\ Harvard University \\ tdeutsch@college.harvard.edu, masoud_jasbiefas.harvard.edu \\ shieber@seas.harvard.edu
}

\begin{abstract}
Readability assessment aims to automatically classify text by the level appropriate for learning readers. Traditional approaches to this task utilize a variety of linguistically motivated features paired with simple machine learning models. More recent methods have improved performance by discarding these features and utilizing deep learning models. However, it is unknown whether augmenting deep learning models with linguistically motivated features would improve performance further. This paper combines these two approaches with the goal of improving overall model performance and addressing this question. Evaluating on two large readability corpora, we find that, given sufficient training data, augmenting deep learning models with linguistically motivated features does not improve state-of-the-art performance. Our results provide preliminary evidence for the hypothesis that the state-of-theart deep learning models represent linguistic features of the text related to readability. Future research on the nature of representations formed in these models can shed light on the learned features and their relations to linguistically motivated ones hypothesized in traditional approaches.
\end{abstract}

\section{Introduction}

Readability assessment poses the task of identifying the appropriate reading level for text. Such labeling is useful for a variety of groups including learning readers and second language learners. Readability assessment systems generally involve analyzing a corpus of documents labeled by editors and authors for reader level. Traditionally, these documents are transformed into a number of linguistic features that are fed into simple models like SVMs and MLPs (Schwarm and Ostendorf, 2005; Vajjala and Meurers, 2012).

More recently, readability assessment models utilize deep neural networks and attention mechanisms (Martinc et al., 2019). While such models achieve state-of-the-art performance on readability assessment corpora, they struggle to generalize across corpora and fail to achieve perfect classification. Often, model performance is improved by gathering additional data. However, readability annotations are time-consuming and expensive given lengthy documents and the need for qualified annotators. A different approach to improving model performance involves fusing the traditional and modern paradigms of linguistic features and deep learning. By incorporating the inductive bias provided by linguistic features into deep learning models, we may be able to reduce the limitations posed by the small size of readability datasets.

In this paper, we evaluate the joint use of linguistic features and deep learning models. We achieve this fusion by simply taking the output of deep learning models as features themselves. Then, these outputs are joined with linguistic features to be further fed into some other model like an SVM. We select linguistic features based on a broad psycholinguistically-motivated composition by Vajjala Balakrishna (2015). Transformers and Hierarchical attention networks were selected as the deep learning models because of their state-ofart performance in readability assessment. Models were evaluated on two of the largest available corpora for readability assessment: WeeBit and Newsela. We also evaluate with different sized training sets to investigate the use of linguistic features in data-poor contexts. Our results find that, given sufficient training data, the linguistic features do not provide a substantial benefit over deep learning methods.

The rest of this paper is organized as follows. Related research is described in section 2. Section 3 details our preprocessing, features, and model construction. Section 4 presents model evaluations on 
two corpora. Section 5 discusses the implications of our results.

We provide a publicly available version of the code used for our experiments. ${ }^{1}$

\section{Related Work}

Work on readability assessment has involved progress on three core components: corpora, features, and models. While early work utilized small corpora, limited feature sets, and simple models, modern research has experimented with a broad set of features and deep learning techniques.

Labeled corpora can be difficult to assemble given the time and qualifications needed to assign a text a readability level. The size of readability corpora expanded significantly with the introduction of the WeeklyReader corpus by Schwarm and Ostendorf (2005). Composed of articles from an educational magazine, the WeeklyReader corpus contains roughly 2,400 articles. The WeeklyReader corpus was then built upon by Vajjala and Meurers (2012) by adding data from the BBC Bitesize website to form the WeeBit corpus. This WeeBit corpus is larger, containing roughly 6,000 documents, while also spanning a greater range of readability levels. Within these corpora, topic and readability are highly correlated. Thus, Xia et al. (2016) constructed the Newsela corpus in which each article is represented at multiple reading levels thereby diminishing this correlation.

Early work on readability assessment, such as that of Flesch (1948), extracted simple textual features like character count. More recently, Schwarm and Ostendorf (2005) analyzed a broader set of features including out-of-vocabulary scores and syntactic features such as average parse tree height. Vajjala and Meurers (2012) assembled perhaps the broadest class of features. They incorporated measures shown by $\mathrm{Lu}$ (2010) to correlate well with second language acquisition measures, as well as psycholinguistically relevant features from the Celex Lexical database and MRC Psycholinguistic Database (Baayen et al., 1995; Wilson, 1988).

Traditional feature formulas, like the Flesch formula, relied on linear models. Later work progressed to more complex related models like SVMs (Schwarm and Ostendorf, 2005). Most recently, state-of-art-performance has been achieved on readability assessment with deep neural network incor-

\footnotetext{
${ }^{1}$ https://github.com/TovlyDeutsch/ Linguistic-Features-for-Readability
}

porating attention mechanisms. These approaches ignore linguistic features entirely and instead feed the raw embeddings of input words, relying on the model itself to extract any relevant features. Specifically, Martinc et al. (2019) found that a pretrained transformer model achieved state-of-the-art performance on the WeeBit corpus while a hierarchical attention network (HAN) achieved state-of-the-art performance on the Newsela corpus.

Deep learning approaches generally exclude any specific linguistic features. In general, a "featureless" approach is sensible given the hypothesis that, with enough data, training, and model complexity, a model should learn any linguistic features that researchers might attempt to precompute. However, precomputed linguistic features may be useful in data-poor contexts where data acquisition is expensive and error-prone. For this reason, in this paper we attempt to incorporate linguistic features with deep learning methods in order to improve readability assessment.

\section{Methodology}

\subsection{Corpora}

\subsubsection{WeeBit}

The WeeBit corpus was assembled by Vajjala and Meurers (2012) by combining documents from the WeeklyReader educational magazine and the BBC Bitesize educational website. They selected classes to assemble a broad range of readability levels intended for readers aged 7 to 16 . To avoid classification bias, they undersampled classes in order to equalize the number of documents in each class to 625. We term this downsampled corpus "WeeBit downsampled". Following the methodologies of Xia et al. (2016) and Martinc et al. (2019), we applied additional preprocessing to the WeeBit corpus in order to remove extraneous material.

\subsubsection{Newsela}

The Newsela corpus (Xia et al., 2016) consists of 1,911 news articles each re-written up to 4 times in simplified manners for readers at different reading levels. This simplification process means that, for any given topic, there exist examples of material on that topic suited for multiple reading levels. This overlap in topic should make the corpus more challenging to label than the WeeBit corpus. In a similar manner to the WeeBit corpus, the Newsela corpus is labeled with grade levels ranging from grade 2 to grade 12. As with WeeBit, these labels 
can either be treated as classes or transformed into numeric labels for regression.

\subsubsection{Labeling Approaches}

Often, readability classes within a corpus are treated as unrelated. These approaches use raw labels as distinct unordered classes. However, readability labels are ordinal, ranging from lower to higher readability. Some work has addressed this issue such as the readability models of Flor et al. (2013) which predict grade levels via linear regression. To test different approaches to acknowledging this ordinality, we devised three methods for labeling the documents: "classification", "age regression", and "ordered class regression".

The classification approach uses the classes originally given. This approach does not suppose any ordinality of the classes. Avoiding such ordinality may be desirable for the sake of simplicity.

"Age regression" applies the mean of the age ranges given by the constituent datasets. For instance, in this approach Level 2 documents from Weekly Reader would be given the label of 7.5 as they are intended for readers of ages 7-8. The advantage of age regression over standard classification is that it provides more precise information about the magnitude of readability differences.

Finally, "ordered class regression" assigns the classes equidistant integers ordered by difficulty. The least difficult class would be labeled " 0 ", the second least difficult class would be labeled " 1 " and so on. As with age regression, this labeling results in a regression rather than classification problem. This method retains the advantage of age regression in demonstrating ordinality. However, ordered regression labeling removes information about the relative differences in difficulty between the classes, instead asserting that they are equidistant in difficulty. The motivation behind this loss of information is that such age differences between classes may not directly translate into differences of difficulty. For instance, the readability difference between documents intended for 7 or 8 yearolds may be much greater than between documents intended for 15 or 16 year-olds because reading development is likely accelerated in younger years.

For final model inferences, we used the classification approach for comparison to previous work. For intermediary CNN models, all three approaches were tested. As the different approaches with $\mathrm{CNN}$ models produced insubstantial differences, other model types were restricted to the simple classifi- cation approach.

\subsection{Features}

Motivated by the success in using linguistic features for modeling readability, we considered a large range of textual analyses relevant to readability. In addition to utilizing features posed in the existing readability research, we investigated formulating new features with a focus on syntactic ambiguity and syntactic diversity. This challenging aspect of language appeared to be underutilized in existing readability literature.

\subsubsection{Existing Features}

To capture a variety of features, we utilized existing linguistic feature computation software ${ }^{2}$ developed by Vajjala Balakrishna (2015) based on 86 feature descriptions in existing readability literature. Given the large number of features, in this section we will focus on the categories of features and their psycholinguistic motivations (where available) and properties. The full list of features used can be found in appendix A.

Traditional Features The most basic features involve what Vajjala and Meurers (2012) refer to as "traditional features" for their use in long-standing readability formulae. They include characters per word, syllables per word, and traditional formulas based on such features like the Flesch-Kincaid formula (Kincaid et al., 1975).

Another set of feature types consists of counts and ratios of part-of-speech tags, extracted using the Stanford parser (Klein and Manning, 2003). In addition to basic parts of speech like nouns, some features include phrase level constituent counts like noun phrases and verb phrases. All of these counts are normalized by either the number of word tokens or number of sentences to make them comparable across documents of differing lengths. These counts are not provided with any psycholinguistic motivation for their use; however, it is not an unreasonable hypothesis that the relative usage of these constituents varies across reading levels. Empirically, these features were shown to have some predictive power for readability. In addition to parts of speech counts, we also utilized word type counts as a simple baseline feature, that is, counting the number of instances of each possible word

\footnotetext{
${ }^{2}$ This code can be found at https://bitbucket. org/nishkalavallabhi/complexity-features.
} 
in the vocabulary. These counts are also divided by document length to generate proportions.

Becoming more abstract than parts of speech, some features count complex syntactic constituent like clauses and subordinated clauses. Specifically, $\mathrm{Lu}$ (2010) found ratios involving sentences, clauses, and t-units ${ }^{3}$ that correlated with second language learners' abilities to read a document. For many of the multi-word syntactic constituents previously described, such as noun phrases and clauses, features were also constructed of their mean lengths. Finally, properties of the syntactic trees themselves were analyzed such as their mean heights.

Moving beyond basic features from syntactic parses, Vajjala Balakrishna (2015) also incorporated "word characteristic" features from linguistic databases. A significant source was the Celex Lexical Database Baayen et al. (1995) which "consists of information on the orthography, phonology, morphology, syntax and frequency for more than 50,000 English lemmas". The database appears to have a focus on morphological data such as whether a word may be considered a loan word and whether it contains affixes. It also contains syntactic properties that may not be apparent from a syntactic parse, e.g. whether a noun is countable. The MRC Psycholinguistic Database Wilson (1988) was also used with a focus on its age of acquisition ratings for words, an clear indicator of the appropriateness of a document's vocabulary.

\subsubsection{Novel Syntactic Features}

We investigated additional syntactic features that may be relevant for readability but whose qualities were not targeted by existing features. These features were used in tandem with the existing linguistic features described previously; future work could utilize these novel feature independently to investigate their particular effect on readability information extraction. For generating syntactic parses, we used the PCFG (probabilistic context-free grammar) parser (Klein and Manning, 2003) from the Stanford Parser package.

Syntactic Ambiguity Sentences can have multiple grammatical syntactic parses. Therefore, syntactic parsers produce multiple parses annotated with parse likelihood. It may seem sensible to use the number of parses generated as a measure of

\footnotetext{
${ }^{3}$ Defined by Vajjala and Meurers (2012) to be "one main clause plus any subordinate clause or non-clausal structure that is attached to or embedded in it".
}

ambiguity. However, this measure is extremely sensitive to sentence length as longer sentences tend to have more possible syntactic parses. Instead, if this list of probabilities is viewed as a distribution, the standard deviation of this distribution is likely to correlate with perceptions of syntactic ambiguity.

\section{Definition 3.1. $P D_{x}$}

The parse deviation, $P D_{x}(s)$, of sentence $s$ is the standard deviation of the distribution of the $x$ most probable parse $\log$ probabilities for $s$. If $s$ has less than $x$ valid parses, the distribution is taken from all the valid parses.

For large values of $x, P D_{x}(s)$ can be significantly sensitive to sentence length: longer sentences are likely to have more valid syntactic parses and thus create low probability tails that increase standard deviation. To reduce this sensitivity, an alternative involves measuring the difference between the largest and mean parse probability.

\section{Definition 3.2. $P D M_{x}$}

$P D M_{x}(s)$ is the difference between the largest parse $\log$ probability and the mean of the log probabilities of the $x$ most probable parses for a sentence s. If $s$ has less than $x$ valid parses, the mean is taken over all the valid parses.

As a compromise between parse investigation and the noise of implausible parses, we selected $P D M_{10}, P D_{10}$, and $P D_{2}$ as features to use in the models of this paper.

Part-of-Speech Divergence To capture the grammatical makeup of a sentence or document, we can count the usage of each part of speech ("POS"), phrase, or clause. The counts can be collected into a distribution. Then, the standard deviation of this distribution, $P O S D_{d e v}$, measures a sentence's grammatical heterogeneity.

\section{Definition 3.3. $P O S D_{d e v}$}

$P O S D_{d e v}(d)$ is the standard deviation of the distribution of POS counts for document $d$.

Similarly, we may want to measure how this grammatical makeup differs from the composition of the document as a whole, a concept that might be termed syntactic uniqueness. To capture this concept, we measure the Kullback-Leibler divergence (Kullback and Leibler, 1951) between the sentence POS count distribution and the document POS count distribution.

Definition 3.4. $P O S_{d i v}$ 
Let $P(s)$ be the distribution of POS counts for sentence $s$ in document $d$. Let $Q$ be the distribution of POS counts for document $d$. Let $|d|$ be the number of sentences in $d$.

$$
\operatorname{POS}_{d i v}(d)=\sum_{s \in d} \frac{D_{K L}(P(s)\|\| Q)}{|d|}
$$

\subsection{Models}

A large range of model complexities were evaluated in order to ascertain the performance improvements, or lack thereof, of additional model complexity. In this section we will describe the specific construction and usage of these models for the experiments conducted in this paper, ordered roughly by model complexity.

\section{SVMs, Linear Models, and Logistic Regression} We used the Scikit-Learn library (Pedregosa et al., 2011) for constructing SVM models. Hyperparameter optimization was performed using the guidelines suggested by Hsu et al. (2003). From the Scikit-Learn library, we also utilized the linear support vector classifier (an SVM with a linear kernel) and logistic regression classifier. As simplicity was the aim for these evaluations, no hyperparameter optimization was performed. The logistic regression classifier was trained using the stochastic average gradient descent ("sag") optimizer.

CNN Convolutional neural networks were selected for their demonstrated performance on sentence classification (Kim, 2014). The CNN model used in this paper is based on the one described by Kim (2014) and implemented using the Keras (Chollet and others, 2015), Tensorflow (Abadi et al., 2015), and Magpie libraries.

Transformer The transformer (Vaswani et al., 2017) is a neural-network-based model that has achieved state-of-the-art results on a wide array of natural language tasks including readability assessment (Martinc et al., 2019). Transformers utilize the mechanism of attention which allows the model to attend to specific parts of the input when constructing the output. Although they are formulated as sequence-to-sequence models, they can be modified to complete a variety of NLP tasks by placing an additional linear layer at the end of the network and training that layer to produce the desired output. This approach often achieves state-of-the-art results when combined with pretraining. In this paper, we use the BERT (Devlin et al., 2019) transformer-based model that is pretrained on BooksCorpus (800M words) (Zhu et al., 2015) and English Wikipedia. The model is then fine-tuned on a specific readability corpus such as WeeBit. The pretrained BERT model is sourced from the Huggingface transformers library (Wolf et al., 2019) and is composed of 12 hidden layers each of size 768 and 12 self-attention heads. The fine-tuning step utilizes an implementation by Martinc et al. (2019). Among the pretrained transformers in the Huggingface library, there are transformers that can accept sequences of size 128, 256, and 512. The 128 sized model was chosen based on the finding by Martinc et al. (2019) that it achieved the highest performance on the WeeBit and Newsela corpora. Documents that exceeded the input sequence size were truncated.

HAN The Hierarchical attention network involves feeding the input through two bidirectional RNNs each accompanied by a separate attention mechanism. One attention mechanism attends to the different words within each sentence while the second mechanism attends to the sentences within the document. These hierarchical attention mechanisms are thought to better mimic the structure of documents and consequently produce superior classification results. The implementation of the model used in this paper is identical to the original architecture described by Yang et al. (2016) and was provided by the authors of Martinc et al. (2019) based on code by Nguyen (2020).

\subsection{Incorporating Linguistic Features with Neural Models}

The neural network models thus far described take either the raw text or word vector embeddings of the text as input. They make no use of linguistic features such as those described in section 3.2. We hypothesized that combining these linguistic features with the deep neural models may improve their performance on readability assessment. Although these models theoretically represent similar features to those prescribed by the linguistic features, we hypothesized that the amount of data and model complexity may be insufficient to capture them. This can be evidenced in certain models failure to generalize across readability corpora. Martinc et al. (2019) found that the BERT model performed well on the WeeBit corpus, achieving a weighted F1 score of 0.8401 , but performed poorly on the Newsela corpus only achieving an F1 
score of 0.5759 . They posit that this disparity occurred "because BERT is pretrained as a language model, [therefore] it tends to rely more on semantic than structural differences during the classification phase and therefore performs better on problems with distinct semantic differences between readability classes". Similarly a HAN was able to achieve better performance than BERT on the Newsela but performed substantially worse on the WeeBit corpus. Thus, under some evaluations the models have deficiencies and fail to generalize. Given these deficiencies, we hypothesized that the inductive bias provided by linguistic features may improve generalizability and overall model performance.

In order to weave together the linguistic features and neural models, we take the simple approach of using the single numerical output of a neural model as a feature itself, joined with linguistic features, and then fed into one of the simpler non-neural models such as SVMs. SVMs were chosen as the final classification model for their simplicity and frequent use in integrating numerical features. The output of the neural model could be any of the label approaches such as grade classes or age regressions described in section 3.1. While all these labeling approaches were tested for CNNs, insubstantial differences in final inferences led us to restrict intermediary results to simple classification for other model types.

\subsection{Training and Evaluation Details}

All experiments involved 5-fold cross validation. All neural-network-based models were trained with the Adam optimizer (Kingma and Ba, 2015) with learning rates of $10^{-3}, 10^{-4}$, and $2^{-5}$ for the $\mathrm{CNN}$, HAN, and transformer respectively. The HAN and CNN models were trained for 20 and 30 epochs. The transformer models were fine-tuned for 3 epochs.

All results are reported as either a weighted $\mathrm{F} 1$ or macro F1 score. To calculate weighted F1, first the $\mathrm{F} 1$ score is calculated for each class independently, as if each class was a case of binary classification. Then, these F1 score are combined in a weighted mean in which each class is weighted by the number of samples in that class. Thus, the weighted F1 score treats each sample equally but prioritizes the most common classes. The macro F1 is similar to the weighted F1 score in that F1 scores are first calculated for each class independently. However, for the macro F1 score, the class F1 scores are com-

\begin{tabular}{lc} 
Features & Weighted F1 \\
\hline HAN & 0.8024 \\
SVM with HAN and linguistic & 0.8014 \\
features & \\
SVM with HAN & 0.7931 \\
SVM with linguistic features and & 0.7694 \\
$\begin{array}{l}\text { Flesch Features } \\
\text { SVM with transformer and lin- }\end{array}$ & 0.7678 \\
guistic features & \\
SVM with transformer, Flesch & 0.7627 \\
features, and linguistic features & \\
SVM with Linguistic features & 0.7582 \\
SVM with CNN age regression & 0.7281 \\
and linguistic features & \\
SVM with CNN ordered classes & 0.7231 \\
regression and linguistic features & \\
SVM with transformer and & 0.7186 \\
Flesch features & \\
\hline $\begin{array}{l}\text { Transformer } \\
\text { CNN }\end{array}$ & 0.5435 \\
\end{tabular}

Table 1: Top 10 performing model results, transformer, and $\mathrm{CNN}$ on the Newsela corpus

bined in a mean without any weighting. Therefore, the macro F1 score treats each class equally but does not treat each sample equally, deprioritizing samples from large classes and prioritizing samples from small classes.

\section{Results}

In this section we report the experimental results of incorporating linguistic features into readability assessment models. The two corpora, WeeBit and Newsela, are analyzed individually and then compared. Our results demonstrate that, given sufficient data, linguistic features provide little to no benefit compared to independent deep learning models. While the corpus experiment results demonstrate a portion of the approaches tested, the full results are available in appendix B

\subsection{Newsela Experiments}

For the Newsela corpus, while linguistic features were able to improve the performance of some models, the top performers did not utilize linguistic features. The results from the top performing models are presented in table 1 .

While the HAN performance was not surpassed by models with linguistic features, the transformer models were. This improvement indicates that lin- 
guistic features capture readability information that transformers cannot capture or have insufficient data to learn. The outsize effect of adding the linguistic features to the transformer models, resulting in a weighted F1 score improvement of 0.22 , may reveal what types of information they address. Martinc et al. (2019) hypothesize that a pretrained language model "tends to rely more on semantic than structural differences" indicating that these features are especially suited to providing non-semantic information such as syntactic qualities.

\subsection{WeeBit Experiments}

The WeeBit corpus was analyzed in two perspectives: the downsampled dataset and the full dataset. Raw results and model rankings were largely comparable between the two dataset sizes.

\subsubsection{Downsampled WeeBit Experiments}

As with the Newsela corpus, the downsampled WeeBit corpus demonstrates no gains from being analyzed with linguistic features. The best performing model, a transformer, did not utilize linguistic features. The results for some of the best performing models are shown in table 2.

Differing with the Newsela corpus, the word type models performed near the top results on the WeeBit corpus comparably to the transformer models. Word type models have no access to word order, thus semantic and topic analysis form their core analysis. Therefore, this result supports the hypothesis of Martinc et al. (2019) that the pretrained transformer is especially attentive to semantic content. This result also indicates that the word type features can provide a significant portion of the information needed for successful readability assessment.

The differing best performing model types between the two corpora are likely due to differing compositions. Unlike the Newsela corpus, the WeeBit corpus shows strong correlation between topic and difficulty. Extracting this topic and semantic content is thought to be a particular strength of the transformer (Martinc et al., 2019) leading to its improved results on this corpus.

\subsubsection{Full WeeBit Experiments}

All of the models were also tested on the full imbalanced WeeBit corpus, the top performing results of which are shown in table 3. Most performance figures increased modestly. However, these gains may not be seen if documents do not match the dis-
Features

Weighted F1

Transformer

0.8387

SVM with transformer, Flesch

0.8381

features, and linguistic features

SVM with transformer and

Flesch features

SVM with transformer and linguistic features

SVM with transformer

Logistic regression classifier

with word types, Flesch features, and linguistic features

Logistic regression classifier

with word types

Logistic regression classifier

with word types, word count, and Flesch features

SVM with CNN classifier and linguistic features

Logistic regression classifier with word types and word count

\begin{tabular}{ll}
\hline \hline CNN & 0.7859 \\
HAN & 0.7507
\end{tabular}

Table 2: Top 10 performing model results, CNN, and HAN on the downsampled WeeBit corpus

tribution of this imbalanced dataset. Additionally, the ranking of models between the downsampled and standard WeeBit corpora showed little change. Although the SVM with transformer and linguistic features performed better than the transformer alone, this difference is extremely small $(<0.005)$ and thus not likely to be statistically significant.

\subsection{Effects of Training Set Size}

One hypothesis explaining the lack of effect of linguistic features is that models learn to extract

\begin{tabular}{lc} 
Features & Weighted F1 \\
\hline $\begin{array}{l}\text { SVM with transformer and lin- } \\
\text { guistic features }\end{array}$ & 0.8769 \\
SVM with transformer and & 0.8746 \\
$\begin{array}{l}\text { Flesch features } \\
\text { SVM with transformer }\end{array}$ & 0.8729 \\
Transformer & 0.8721 \\
SVM with transformer, Flesch & 0.8721 \\
features, and linguistic features &
\end{tabular}

Table 3: Top 5 performing model results on the WeeBit corpus 


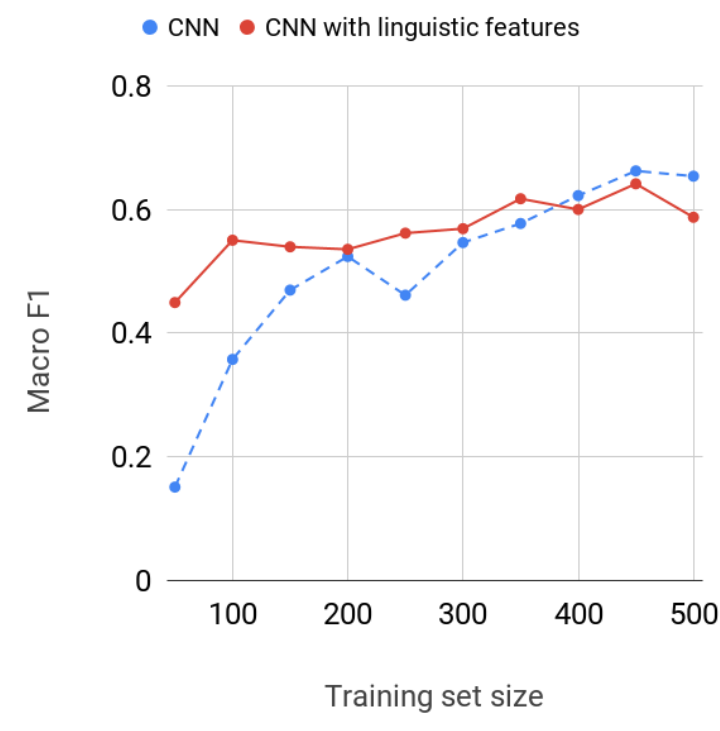

Figure 1: Performance differences across different training set sizes on the downsampled WeeBit corpus

those features given enough data. Thus, perhaps in more data-poor environments the linguistic features would prove more useful. To test this hypothesis, we evaluated two CNN-based models, one with linguistic features and one without, with various sized training subsets of the downsampled WeeBit corpus. The macro F1 at these various dataset sizes is shown in figure 1. Across the trials at different training set sizes, the test set is held constant thereby isolating the impact of training set size.

The hypothesis holds true for extremely small subsets of training data, those with fewer than 200 documents. Above this training set size, the addition of linguistic features results in insubstantial changes in performance. Thus, either the patterns exposed by the linguistic features are learnable with very little data or the patterns extracted by deep learning models differ significantly from the linguistic features. The latter appears more likely given that linguistic features are shown to improve performance for certain corpora (Newsela) and model types (transformers).

This result indicates that the use of linguistic features should be considered for small datasets. However, the dataset size at which those features lose utility is extremely small. Therefore, collecting additional data would often be more efficient than investing the time to incorporate linguistic features.

\subsection{Effects of Linguistic Features}

Overall, the failure of linguistic features to improve state-of-the-art deep learning models indicates that, given the available corpora, model complexity, and model structures, they do not add information over and beyond what the state-of-the-art models have already learned. However, in certain data-poor contexts, they can improve the performance of deep learning models. Similarly, with more diverse and more accurately and consistently labeled corpora, the linguistic features could prove more useful. It may be the case that the best performing models already achieve near the maximal possible performance on this corpus. The reason the maximal performance may be below a perfect score (an F1 score of 1) is disagreement and inconsistency in dataset labeling. Presumably the dataset was assessed by multiple labelers who may not have always agreed with one another or even with themselves. Thus, if either a new set of human labelers or the original labelers are tasked with labeling readability in this corpus, they may only achieve performance similar to the best performance seen in these experiments. Performing this human experiment would be a useful analysis of corpus validity and consistency. Similarly, a more diverse corpus (differing in length, topic, writing style, etc.) may prove more difficult for the models to label alone without additional training data; in this case, the linguistic features may prove more helpful in providing inductive bias.

Additionally, the lack of improvement from adding linguistic features indicates that deep learning models may already be representing those features. Future work could probe the models for different aspects of the linguistic features, thereby investigating what properties are most relevant for readability.

\section{Conclusion}

In this paper we explored the role of linguistic features in deep learning methods for readability assessment, and asked: can incorporating linguistic features improve state-of-the-art models? We constructed linguistic features focused on syntactic properties ignored by existing features. We incorporated these features into a variety of model types, both those commonly used in readability research and more modern deep learning methods. We evaluated these models on two distinct corpora that posed different challenges for readability assess- 
ment. Additional evaluations were performed with various training set sizes to explore the inductive bias provided by linguistic features. While linguistic features occasionally improved model performance, particularly at small training set sizes, these models did not achieve state-of-the-art performance.

Given that linguistic features did not generally improve deep learning models, these models may be already implicitly capturing the features that are useful for readability assessment. Thus, future work should investigate to what degree the models represent linguistic features, perhaps via probing methods.

Although this work supports disusing linguistic features in readability assessment, this assertion is limited by available corpora. Specifically, ambiguity in the corpora construction methodology limits our ability to measure label consistency and validity. Therefore, the maximal possible performance may already be achieved by state-of-the-art models. Thus, future work should explore constructing and evaluating readability corpora with rigorous consistent methodology; such corpora may be assessed most effectively using linguistic features. For instance, accuracy could be improved by averaging across multiple labelers.

Overall, linguistic features do not appear to be useful for readability assessment. While often used in traditional readability assessment models, these features generally fail to improve the performance of deep learning methods. Thus, this paper provides a starting point to understanding the qualities and abilities of deep learning models in comparison to linguistic features. Through this comparison, we can analyze what types of information these models are well-suited to learning.

\section{References}

Martín Abadi, Ashish Agarwal, Paul Barham, Eugene Brevdo, Zhifeng Chen, Craig Citro, Greg S. Corrado, Andy Davis, Jeffrey Dean, Matthieu Devin, Sanjay Ghemawat, Ian Goodfellow, Andrew Harp, Geoffrey Irving, Michael Isard, Yangqing Jia, Rafal Jozefowicz, Lukasz Kaiser, Manjunath Kudlur, Josh Levenberg, Dandelion Mané, Rajat Monga, Sherry Moore, Derek Murray, Chris Olah, Mike Schuster, Jonathon Shlens, Benoit Steiner, Ilya Sutskever, Kunal Talwar, Paul Tucker, Vincent Vanhoucke, Vijay Vasudevan, Fernanda Viégas, Oriol Vinyals, Pete Warden, Martin Wattenberg, Martin Wicke, Yuan Yu, and Xiaoqiang Zheng. 2015. TensorFlow: large-scale ma- chine learning on heterogeneous systems. Technical report.

Rolf Harald Baayen, Richard Piepenbrock, and Leon Gulikers. 1995. The CELEX lexical database.

François Chollet and others. 2015. Keras.

Jacob Devlin, Ming-Wei Chang, Kenton Lee, and Kristina Toutanova. 2019. BERT: Pre-training of Deep Bidirectional Transformers for Language Understanding. In Proceedings of the 2019 Conference of the North American Chapter of the Association for Computational Linguistics: Human Language Technologies, Volume 1 (Long and Short Papers), pages 4171-4186, Minneapolis, Minnesota. Association for Computational Linguistics.

Rudolph Flesch. 1948. A new readability yardstick. Journal of Applied Psychology, 32(3):221-233.

Michael Flor, Beata Beigman Klebanov, and Kathleen M. Sheehan. 2013. Lexical tightness and text complexity. In Proceedings of the Workshop on Natural Language Processing for Improving Textual Accessibility, pages 29-38, Atlanta, Georgia. Association for Computational Linguistics.

Chih-wei Hsu, Chih-chung Chang, and Chih-Jen Lin. 2003. A practical guide to support vector classification. Technical report.

Yoon Kim. 2014. Convolutional Neural Networks for Sentence Classification. In Proceedings of the 2014 Conference on Empirical Methods in Natural Language Processing (EMNLP), pages 1746-1751, Doha, Qatar. Association for Computational Linguistics.

J. P. Kincaid, Jr. Fishburne, Rogers Robert P., Chissom Richard L., and Brad S. 1975. Derivation of New Readability Formulas (Automated Readability Index, Fog Count and Flesch Reading Ease Formula) for Navy Enlisted Personnel:. Technical report, Defense Technical Information Center, Fort Belvoir, VA.

Diederik P. Kingma and Jimmy Ba. 2015. Adam: A method for stochastic optimization. In 3rd International Conference on Learning Representations, ICLR 2015, San Diego, CA, USA, May 7-9, 2015, Conference Track Proceedings.

Dan Klein and Christopher D. Manning. 2003. Accurate unlexicalized parsing. In Proceedings of the 41st Annual Meeting on Association for Computational Linguistics - ACL '03, volume 1, pages 423430, Sapporo, Japan. Association for Computational Linguistics.

Solomon Kullback and Richard A. Leibler. 1951. On Information and Sufficiency. The Annals of Mathematical Statistics, 22(1):79-86. 
Victor Kuperman, Hans Stadthagen-Gonzalez, and Marc Brysbaert. 2012. Age-of-acquisition ratings for 30,000 English words. Behavior Research Methods, 44(4):978-990.

Xiaofei Lu. 2010. Automatic analysis of syntactic complexity in second language writing. International Journal of Corpus Linguistics, 15(4):474-496.

Matej Martinc, Senja Pollak, and Marko RobnikŠikonja. 2019. Supervised and unsupervised neural approaches to text readability. Computing Research Repository, arXiv:1503.06733. Version 2.

Viet Nguyen. 2020. Hierarchical Attention Networks for Document Classification. Original-date: 201901-31T18:56:40Z.

Fabian Pedregosa, Gaël Varoquaux, Alexandre Gramfort, Vincent Michel, Bertrand Thirion, Olivier Grisel, Mathieu Blondel, Peter Prettenhofer, Ron Weiss, Vincent Dubourg, Jake Vanderplas, Alexandre Passos, David Cournapeau, Matthieu Brucher, Matthieu Perrot, and Édouard Duchesnay. 2011. Scikit-learn: Machine Learning in Python. Journal of Machine Learning Research, 12:2825-2830.

Sarah E. Schwarm and Mari Ostendorf. 2005. Reading Level Assessment Using Support Vector Machines and Statistical Language Models. In Proceedings of the 43rd Annual Meeting on Association for Computational Linguistics, ACL '05, pages 523-530, Stroudsburg, PA, USA. Association for Computational Linguistics. Event-place: Ann Arbor, Michigan.

Sowmya Vajjala and Detmar Meurers. 2012. On Improving the Accuracy of Readability Classification using Insights from Second Language Acquisition. In Proceedings of the Seventh Workshop on Building Educational Applications Using NLP, pages 163173, Montréal, Canada. Association for Computational Linguistics.

Sowmya Vajjala Balakrishna. 2015. Analyzing Text Complexity and Text Simplification: Connecting Linguistics, Processing and Educational Applications. Dissertation, Universität Tübingen.

Ashish Vaswani, Noam Shazeer, Niki Parmar, Jakob Uszkoreit, Llion Jones, Aidan N Gomez, Łukasz Kaiser, and Illia Polosukhin. 2017. Attention is all you need. In Advances in neural information processing systems 30, pages 5998-6008. Curran Associates, Inc.

Michael Wilson. 1988. MRC psycholinguistic database: Machine-usable dictionary, version 2.00 Behavior Research Methods, Instruments, \& Computers, 20(1):6-10.

Thomas Wolf, Lysandre Debut, Victor Sanh, Julien Chaumond, Clement Delangue, Anthony Moi, Pierric Cistac, Tim Rault, R'emi Louf, Morgan Funtowicz, and Jamie Brew. 2019. HuggingFace's transformers: state-of-the-art natural language processing. Technical report.
Menglin Xia, Ekaterina Kochmar, and Ted Briscoe. 2016. Text Readability Assessment for Second Language Learners. In Proceedings of the 11th Workshop on Innovative Use of NLP for Building Educational Applications, pages 12-22, San Diego, CA. Association for Computational Linguistics.

Zichao Yang, Diyi Yang, Chris Dyer, Xiaodong He, Alex Smola, and Eduard Hovy. 2016. Hierarchical Attention Networks for Document Classification. In Proceedings of the 2016 Conference of the North American Chapter of the Association for Computational Linguistics: Human Language Technologies, pages 1480-1489, San Diego, California. Association for Computational Linguistics.

Yukun Zhu, Ryan Kiros, Richard Zemel, Ruslan Salakhutdinov, Raquel Urtasun, Antonio Torralba, and Sanja Fidler. 2015. Aligning Books and Movies: Towards Story-like Visual Explanations by Watching Movies and Reading Books. Computing Research Repository, arXiv:1506.06724. 


\section{A Feature Definitions}

For the following definitions, if the a ratio is undefined (i.e. the denominator is zero) the result is treated as zero. Vajjala and Meurers (2012) define complex nominals to be: "a) nouns plus adjective, possessive, prepositional phrase, relative clause, participle or appositive, b) nominal clauses, c) gerunds and infinitives in subject positions." Here polysyllabic means more than two syllables and "long words" means a word with seven or more characters. Descriptions of the norms of age of acquisition ratings can be found in Kuperman et al. (2012).

\begin{tabular}{|c|l|}
\hline Feature Name & Definition \\
\hline$P D_{x}(s)$ & $\begin{array}{l}\text { The parse deviation, } P D_{x}(s) \text {, of sentence } s \text { is the standard deviation of the distribution } \\
\text { of the } x \text { most probable parse log probabilities for } s . \text { If } s \text { has less than } x \text { valid parses, } \\
\text { the distribution is taken from all the valid parses. }\end{array}$ \\
\hline$P D M_{x}$ & $\begin{array}{l}P D M_{x}(s) \text { is the difference between the largest parse log probability and the mean of } \\
\text { the log probabilities of the } x \text { most probable parses for a sentence s. If } s \text { has less than } \\
x \text { valid parses, the mean is taken over all the valid parses. }\end{array}$ \\
\hline$P O S D_{d e v}$ & $\begin{array}{l}P O S D_{d e v}(d) \text { is the standard deviation of the distribution of POS counts for document } \\
d .\end{array}$ \\
\hline$P O S_{d i v}$ & $\begin{array}{l}\text { Let } P(s) \text { be the distribution of POS counts for sentence } s \text { in document } d \text {. Let } Q \text { be } \\
\text { the distribution of POS counts for document } d . \text { Let }|d| \text { be the number of sentences in } \\
d . P O S_{d i v}(d)=\sum_{s \in d} \frac{D_{K L}(P(s)\|\| \|)}{|d|}\end{array}$ \\
\hline
\end{tabular}

Table 4: Novel syntactic feature definitions 


\begin{tabular}{|c|l|}
\hline Feature Name & Definition \\
\hline mean t-unit lenght & number of words / number of t-units \\
\hline mean parse tree height per sentence & mean parse tree height / number of sentences \\
\hline subtrees per sentence & number of subtrees / number of sentences \\
\hline SBARs per sentence & number of SBARs / number of sentences \\
\hline NPs per sentence & number of NPs / number of sentences \\
\hline VPs per sentence & number of VPs / number of sentences \\
\hline PPs per sentence & number of PPs / number of sentences \\
\hline mean NP size & number of children of NPs / number of NPs \\
\hline mean VP size & number of children of VPs / number of VPs \\
\hline mean PP size & number of children of PPs / number of PPs \\
\hline WHPs per sentence & number of wh-phrases / number of sentences \\
\hline RRCs per sentence & number of reduced relative clauses / number of sentences \\
\hline ConjPs per sentence & number of conjunction phrases / number of sentences \\
\hline clauses per sentence & number of clauses / number of sentences \\
\hline t-units per sentence & number of t-units / number of sentences \\
\hline clauses per t-unit & number of clauses / number of t-units \\
\hline complex t-unit ratio & number of t-units that contain a dependent clause / number of t-units \\
\hline dependent clauses per clause & number of dependent clauses / number of clauses \\
\hline dependent clauses per t-unit & number of dependent clauses / number of t-units \\
\hline coordinate clauses per clause & number of coordinate clauses / number of clauses \\
\hline coordinate clauses per t-unit & number of coordinate clauses / number of t-units \\
\hline complex nominals per clauses & number of complex nominals / number of clauses \\
\hline complex nominals per t-unit & number of complex nominals / number of t-units \\
\hline VPs per t-unit & number of VP / number of t-units \\
\hline &
\end{tabular}

Table 5: Existing syntactic-parse-based feature definitions 


\begin{tabular}{|c|l|}
\hline Feature Name & Definition \\
\hline nouns per word & number of nouns / number of words \\
\hline proper nouns per word & number of proper nouns / number of words \\
\hline pronouns per word & number of pronouns / number of words \\
\hline conjuctions per word & number of conjuctions / number of words \\
\hline adjectives per word & number of adjectives / number of words \\
\hline verbs per word & number of verbs / number of words \\
\hline adverbs per word & number of adverbs / number of words \\
\hline modal verbs per word & number of modal verbs / number of words \\
\hline prepositions per word & number of prepositions / number of words \\
\hline interjections per word & number of interjections / number of words \\
\hline personal pronouns per word & number of personal pronouns / number of words \\
\hline wh-pronouns per word & number of wh-pronouns / number of words \\
\hline lexical words per word & number of lexical words / number of words \\
\hline function words per word & number of function words / number of words \\
\hline determiners per word & number of determiners / number of words \\
\hline VBs per word & number of base form verbs / number of words \\
\hline VBDs per word & number of past tense verbs / number of words \\
\hline VBGs per word & number of gerund or present participle verbs / number of words \\
\hline VBNs per word & number of past participle verbs / number of words \\
\hline VBPs per word & number of non-3rd person singular present verbs / number of \\
& words \\
\hline VBZs per word & number of 3rd person singular present verbs / number of words \\
\hline adverb variation & number of adverbs / number of lexical words \\
\hline adjective variation & number of adjectives / number of lexical words \\
\hline modal verb variation & number of adverbs and adverbs / number of lexical words \\
\hline noun variation & number of nouns / number of lexical words \\
\hline verb variation-I & number of verbs / number of unique verbs \\
\hline verb variation-II & number of verbs / number of lexical words \\
\hline squared verb variation-I & (number of verbs $)^{2} /$ number of unique verbs \\
\hline corrected verb variation-I & number of verbs / $\sqrt{2} *$ number of unique verbs \\
\hline
\end{tabular}

Table 6: Existing POS-tag-based feature definitions

\begin{tabular}{|c|l|}
\hline Feature Name & Definition \\
\hline AoA Kuperman & Mean age of acquisition of words (Kuperman database) \\
\hline AoA Kuperman lemmas & Mean age of acquisition of lemmas \\
\hline AoA Bird lemmas & Mean age of acquisition of lemmas, Bird norm \\
\hline AoA Bristol lemmas & Mean age of acquisition of lemmas, Bristol norm \\
\hline AoA Cortese and Khanna lemmas & Mean age of acquisition of lemmas, Cortese and Khanna norm \\
\hline MRC familiarity & Mean word familiarity rating \\
\hline MRC concreteness & Mean word concreteness rating \\
\hline MRC Imageability & Mean word imageability rating \\
\hline MRC Colorado Meaningfulness & mean word Colorado norms meaningfulness rating \\
\hline MRC Pavio Meaningfulness & mean word Pavio norms meaningfulness rating \\
\hline MRC AoA & Mean age of acquisition of words (MRC database) \\
\hline
\end{tabular}

Table 7: Existing psycholinguistic feature definitions 


\begin{tabular}{|c|l|}
\hline Feature Name & Definition \\
\hline number of sentences & number of sentences \\
\hline mean sentence length & number of words / number of sentences \\
\hline number of characters & number of characters \\
\hline number of syllables & number of syllables \\
\hline Flesch-Kincaid Formula & $11.8 *$ syllables per word $+0.39 *$ words per sentence -15.59 \\
\hline Flesch Fomula & $206.835-1.015 *$ words per sentence $-84.6 *$ syllables per word \\
\hline Automated Readability Index & $4.71 *$ characters per word $+0.5 *$ words per sentence -21.43 \\
\hline Coleman Liau Formula & $-29.5873 *$ sentences per word $+5.8799 *$ characters per word -15.8007 \\
\hline SMOG Formula & $1.0430 * \sqrt{30.0} *$ polysyllabic words per sentence +3.1291 \\
\hline Fog Fomula & $($ words per sentence + proportion of words that are polysylabic $) * 0.4$ \\
\hline FORCAST Readability Formula & $20-15 *$ monosylabic words per word \\
\hline LIX Readability Formula & words per sentence + long words perword $* 100.0$ \\
\hline
\end{tabular}

Table 8: Existing traditional feature definitions

\begin{tabular}{|c|l|}
\hline Feature Name & Definition \\
\hline type token ratio & number of word types / number of word tokens \\
\hline corrected type token ratio & number of word types $/ \sqrt{2 * \text { number of word tokens }}$ \\
\hline root type token ratio & number of word types $/ \sqrt{\text { number of word tokens }}$ \\
\hline bilogorathmic type token ratio & $\log ($ number of word types $) / \log ($ number of word tokens $)$ \\
\hline uber index & $(\log (\text { number of word types }))^{2} / \log \left(\frac{\text { number of word tokens }}{\text { number of word types }}\right)$ \\
\hline measure of textual lexical diversity (MTLD) & see McCarthy and Jarvis, 2010 \\
\hline number of senses & total number of senses across all words / number of word tokens \\
\hline hyeprnyms per word & number of hypernyms / number of word tokens \\
\hline hyponyms per word & total number of senses hyponyms / number of word tokens \\
\hline
\end{tabular}

Table 9: Existing traditional feature definitions 


\section{B Full Model Results}

Features

$\begin{array}{llll}\text { Weighted } & \text { Macro F1 } & \text { SD } & \text { SD macro } \\ \text { F1 } & & \text { weighted } & \text { F1 } \\ & & \text { F1 } & \end{array}$

\begin{tabular}{|c|c|c|c|c|}
\hline Linear classifier with Flesch Score & 0.2147 & 0.2156 & 0.0347 & 0.0253 \\
\hline Linear classifier with Flesch features & 0.3973 & 0.3976 & 0.0154 & 0.0087 \\
\hline SVM with HAN & 0.5531 & 0.5499 & 0.1944 & 0.1928 \\
\hline SVM with Flesch features & 0.5908 & 0.5905 & 0.0157 & 0.0168 \\
\hline SVM with CNN ordered class regression & 0.6703 & 0.6700 & 0.0360 & 0.0334 \\
\hline SVM with CNN age regression & 0.6743 & 0.6742 & 0.0339 & 0.0314 \\
\hline Linear classifier with word types & 0.7202 & 0.7189 & 0.0063 & 0.0085 \\
\hline $\begin{array}{l}\text { SVM with CNN ordered classes regression, } \\
\text { and linguistic features }\end{array}$ & 0.7265 & 0.7262 & 0.0326 & 0.0297 \\
\hline $\begin{array}{l}\text { Logistic regression classification with word } \\
\text { types, Flesch features, and linguistic features }\end{array}$ & 0.7382 & 0.7376 & 0.0710 & 0.0684 \\
\hline $\begin{array}{l}\text { SVM with CNN age regression and linguistic } \\
\text { features }\end{array}$ & 0.7384 & 0.7376 & 0.0361 & 0.0346 \\
\hline HAN & 0.7507 & 0.7501 & 0.0306 & 0.0302 \\
\hline $\begin{array}{l}\text { SVM with linguistic features and Flesch fea- } \\
\text { tures }\end{array}$ & 0.7664 & 0.7667 & 0.0109 & 0.0114 \\
\hline SVM with linguistic features & 0.7665 & 0.7666 & 0.0146 & 0.0153 \\
\hline $\mathrm{CNN}$ & 0.7859 & 0.7852 & 0.0171 & 0.0166 \\
\hline SVM with HAN and linguistic features & 0.7862 & 0.7864 & 0.0631 & 0.0633 \\
\hline SVM with CNN classifier & 0.7882 & 0.7879 & 0.0217 & 0.0195 \\
\hline Logistic regression with word types & 0.7894 & 0.7887 & 0.0151 & 0.0202 \\
\hline $\begin{array}{l}\text { Logistic regression classification with word } \\
\text { types and word count }\end{array}$ & 0.7908 & 0.7899 & 0.0130 & 0.0182 \\
\hline $\begin{array}{l}\text { SVM with CNN classifier and linguistic fea- } \\
\text { tures }\end{array}$ & 0.7923 & 0.7919 & 0.0210 & 0.0193 \\
\hline $\begin{array}{l}\text { Logistic regression classification with word } \\
\text { types, word count, and Flesch features }\end{array}$ & 0.7934 & 0.7926 & 0.0135 & 0.0187 \\
\hline $\begin{array}{l}\text { Logistic regression with word types, Flesch } \\
\text { features, and linguistic features }\end{array}$ & 0.8135 & 0.8130 & 0.0131 & 0.0169 \\
\hline SVM with transformer & 0.8343 & 0.8340 & 0.0131 & 0.0135 \\
\hline SVM with transformer and linguistic features & 0.8344 & 0.8347 & 0.0106 & 0.0091 \\
\hline SVM with transformer and Flesch features & 0.8359 & 0.8358 & 0.0151 & 0.0154 \\
\hline $\begin{array}{l}\text { SVM with transformer, Flesch features, and } \\
\text { linguistic features }\end{array}$ & 0.8381 & 0.8377 & 0.0128 & 0.0118 \\
\hline Transformer & 0.8387 & 0.8388 & 0.0097 & 0.0073 \\
\hline
\end{tabular}

Table 10: WeeBit downsampled model results sorted by weighted F1 score 
Features

Weighted Macro F1 SD SD F1 weighted Macro F1

F1

\begin{tabular}{|c|c|c|c|c|}
\hline Linear classifier with Flesch Score & 0.3357 & 0.1816 & 0.0243 & 0.0079 \\
\hline SVM with HAN & 0.3625 & 0.2134 & 0.0400 & 0.0331 \\
\hline Linear classifier with Flesch features & 0.3939 & 0.2639 & 0.0239 & 0.0305 \\
\hline SVM with Flesch features & 0.4776 & 0.3609 & 0.0222 & 0.0190 \\
\hline SVM with CNN age regression & 0.7279 & 0.6431 & 0.0198 & 0.0205 \\
\hline SVM with CNN ordered class regression & 0.7316 & 0.6482 & 0.0142 & 0.0141 \\
\hline $\begin{array}{l}\text { SVM with CNN age regression and linguistic } \\
\text { features }\end{array}$ & 0.7779 & 0.7088 & 0.0156 & 0.0194 \\
\hline $\begin{array}{l}\text { SVM with CNN ordered classes regression, } \\
\text { and linguistic features }\end{array}$ & 0.7797 & 0.7114 & 0.0130 & 0.0120 \\
\hline Linear classifier with word types & 0.7821 & 0.7109 & 0.0162 & 0.0127 \\
\hline $\begin{array}{l}\text { SVM with Linguistic features and Flesch fea- } \\
\text { tures }\end{array}$ & 0.7952 & 0.7367 & 0.0121 & 0.0157 \\
\hline SVM with Linguistic features & 0.7952 & 0.7366 & 0.0130 & 0.0164 \\
\hline HAN & 0.8065 & 0.7435 & 0.0123 & 0.0220 \\
\hline $\begin{array}{l}\text { Logistic regression classification with word } \\
\text { types }\end{array}$ & 0.8088 & 0.7497 & 0.0127 & 0.0152 \\
\hline $\begin{array}{l}\text { Logistic regression classification with word } \\
\text { types and word count }\end{array}$ & 0.8088 & 0.7497 & 0.0121 & 0.0148 \\
\hline $\begin{array}{l}\text { Logistic regression classification with word } \\
\text { types, word count, and Flesch features }\end{array}$ & 0.8098 & 0.7505 & 0.0130 & 0.0163 \\
\hline $\begin{array}{l}\text { Logistic regression classification with word } \\
\text { types, Flesch features, and linguistic features }\end{array}$ & 0.8206 & 0.7664 & 0.0428 & 0.0500 \\
\hline $\mathrm{CNN}$ & 0.8282 & 0.7748 & 0.0211 & 0.0183 \\
\hline $\begin{array}{l}\text { SVM with CNN classifier and linguistic fea- } \\
\text { tures }\end{array}$ & 0.8286 & 0.7753 & 0.0222 & 0.0209 \\
\hline $\begin{array}{l}\text { Logistic regression classification with word } \\
\text { types, Flesch features, and ling features }\end{array}$ & 0.8293 & 0.7760 & 0.0152 & 0.0172 \\
\hline SVM with CNN classifier & 0.8296 & 0.7754 & 0.0163 & 0.0136 \\
\hline SVM with HAN and linguistic features & 0.8441 & 0.7970 & 0.0643 & 0.0827 \\
\hline $\begin{array}{l}\text { SVM with transformer, Flesch features, and } \\
\text { linguistic features }\end{array}$ & 0.8721 & 0.8273 & 0.0095 & 0.0121 \\
\hline Transformer & 0.8721 & 0.8272 & 0.0071 & 0.0102 \\
\hline SVM with transformer & 0.8729 & 0.8288 & 0.0064 & 0.0090 \\
\hline SVM with transformer and Flesch features & 0.8746 & 0.8305 & 0.0054 & 0.0107 \\
\hline SVM with transformer and linguistic features & 0.8769 & 0.8343 & 0.0077 & 0.0129 \\
\hline
\end{tabular}

Table 11: WeeBit model results sorted by weighted F1 score 
Features

Weighted Macro F1 SD SD

F1 weighted Macro F1

F1

\begin{tabular}{|c|c|c|c|c|}
\hline Linear classifier with Flesch Score & 0.1668 & 0.0915 & 0.0055 & 0.0043 \\
\hline SVM with Flesch score & 0.2653 & 0.1860 & 0.0053 & 0.0086 \\
\hline Logistic regression with word types & 0.2964 & 0.2030 & 0.0144 & 0.0103 \\
\hline $\begin{array}{l}\text { Logistic regression with word types and word } \\
\text { count }\end{array}$ & 0.2969 & 0.2039 & 0.0145 & 0.0095 \\
\hline $\begin{array}{l}\text { Logistic regression with word types, word } \\
\text { count, and Flesch features }\end{array}$ & 0.3006 & 0.2097 & 0.0139 & 0.0088 \\
\hline Linear classifier with Flesch features & 0.3080 & 0.2060 & 0.0110 & 0.0077 \\
\hline $\begin{array}{l}\text { Logistic regression with word types, Flesch } \\
\text { features, and linguistic features }\end{array}$ & 0.3333 & 0.2489 & 0.0118 & 0.0162 \\
\hline Linear classifier with word types & 0.3368 & 0.2485 & 0.0089 & 0.0153 \\
\hline $\mathrm{CNN}$ & 0.3379 & 0.2574 & 0.0038 & 0.0111 \\
\hline SVM with CNN classifier & 0.3407 & 0.2616 & 0.0079 & 0.0142 \\
\hline SVM with CNN ordered class regression & 0.5207 & 0.4454 & 0.0092 & 0.0193 \\
\hline SVM with CNN age regression & 0.5223 & 0.4469 & 0.0149 & 0.0244 \\
\hline SVM with transformer & 0.5430 & 0.4711 & 0.0095 & 0.0258 \\
\hline Transformer & 0.5435 & 0.4713 & 0.0106 & 0.0264 \\
\hline Linear classifier with linguistic features & 0.5573 & 0.4748 & 0.0053 & 0.0140 \\
\hline $\begin{array}{l}\text { SVM with CNN classifier, and linguistic fea- } \\
\text { tures }\end{array}$ & 0.7058 & 0.5510 & 0.0079 & 0.0357 \\
\hline SVM with Flesch features & 0.7177 & 0.6257 & 0.0079 & 0.0292 \\
\hline SVM with transformer and Flesch features & 0.7186 & 0.6305 & 0.0074 & 0.0282 \\
\hline $\begin{array}{l}\text { SVM with CNN ordered classes regression } \\
\text { and linguistic features }\end{array}$ & 0.7231 & 0.6053 & 0.0062 & 0.0331 \\
\hline $\begin{array}{l}\text { SVM with CNN age regression and linguistic } \\
\text { features }\end{array}$ & 0.7281 & 0.6104 & 0.0057 & 0.0337 \\
\hline SVM with linguistic features & 0.7582 & 0.6432 & 0.0089 & 0.0379 \\
\hline $\begin{array}{l}\text { SVM with transformer, Flesch features, and } \\
\text { linguistic features }\end{array}$ & 0.7627 & 0.6263 & 0.0075 & 0.0301 \\
\hline SVM with transformer and linguistic features & 0.7678 & 0.6656 & 0.0230 & 0.0385 \\
\hline $\begin{array}{l}\text { SVM with linguistic features and Flesch Fea- } \\
\text { tures }\end{array}$ & 0.7694 & 0.6446 & 0.0060 & 0.0406 \\
\hline SVM with HAN & 0.7931 & 0.6724 & 0.0448 & 0.0449 \\
\hline SVM with HAN and linguistic features & 0.8014 & 0.6751 & 0.0263 & 0.0379 \\
\hline HAN & 0.8024 & 0.6775 & 0.1116 & 0.1825 \\
\hline
\end{tabular}

Table 12: Newsela model results sorted by weighted F1 score 